\title{
Identification of novel Ralstonia solanacearum type III effector proteins through translocation analysis of hrpB-regulated gene products
}

\author{
Correspondence \\ Takafumi Mukaihara \\ mukaihara@bio-ribs.com
}

Received 22 January 2009

Revised 6 April 2009

Accepted 24 April 2009
Takafumi Mukaihara and Naoyuki Tamurat

Research Institute for Biological Sciences, Okayama (RIBS), 7549-1 Yoshikawa, Kibichuo-cho, Okayama 716-1241, Japan

\section{INTRODUCTION}

Ralstonia solanacearum is a Gram-negative soilborne pathogen that causes bacterial wilt in more than 100 plant species, including agriculturally important crops such as tomato, potato, tobacco, banana and eggplant (Hayward, 1991). This pathogen enters plant roots from natural openings or wounds, invades xylem vessels, proliferates and spreads throughout the vascular system, and secretes a large amount of extracellular polysaccharides (EPSs) that impair water transport, resulting in the lethal wilting of infected plants (Schell, 2000).

The type III secretion system (TTSS) is essential for the pathogenicity of many plant- and animal-pathogenic bacteria (Cornelis \& Van Gijsegem, 2000; Galán \& Collmer, 1999; He et al., 2004). Some components of the TTSS are similar to those of the flagellar basal body (Gophna et al., 2003). In fact, the TTSS machinery complexes isolated from several animal-pathogenic bacteria, such as Salmonella typhimurium, Shigella flexneri and

tPresent address: Agricultural Experimental Station, Okayama Prefectural General Agriculture Center, 1174-1 Koda-Oki, Akaiwa 709-0801, Japan.

Abbreviations: EPS, extracellular polysaccharide; LRR, leucine-rich repeat; PIP, plant-inducible promoter; TTSS, type III secretion system.

A supplementary table of primers and plasmids is available with the online version of this paper. enteropathogenic Escherichia coli, have been shown to form a cylindrical structure, similar to a flagellar basal body, which is composed of outer and inner rings, an inner rod that traverses the two rings and a needle-like projection (Galán \& Wolf-Watz, 2006). In plant pathogens, the TTSS is called the Hrp (hypersensitive response and pathogenicity) because defects in the TTSS result in the loss of both the ability to induce hypersensitive responses and pathogenicity in plants (Alfano \& Collmer, 1997). A long filamentous extracellular appendage associated with the Hrp TTSS, called the Hrp pilus, has been found (Jin \& He, 2001; Roine et al., 1997; Van Gijsegem et al., 2000; Weber, et al., 2005). These TTSSs are considered to act as molecular syringes or conduits for injecting virulence factors, called effector proteins, into host cells, where they suppress or stimulate a variety of host cellular functions to promote the growth of the pathogen (Alfano \& Collmer, 2004; Jones \& Dangle, 2006; Mudgett, 2005; Nomura et al., 2005).

Various approaches have been taken to identify type III effector proteins in plant-pathogenic bacteria. Genes belonging to the hrp regulon have been isolated as candidate effector genes by genetic screening (Fouts et al., 2002; Noël et al., 2001), by analysing specific promoter sequences in genome DNA sequences (Cunnac et al., 2004; Zwiesler-Vollick et al., 2002) or by whole-genomic microarray analyses (Ferreira et al., 2006; Occhialini et al., 
2005) because the expression of many effector genes is coregulated with that of $h r p$ genes by common regulatory proteins in many plant pathogens. The $\mathrm{N}$-terminal export signals of effector proteins have also been used as a characteristic to survey candidate effector genes in genomic DNA sequences (Petnicki-Ocwieja et al., 2002). True effector proteins were selected from these candidates by testing their ability to be translocated into plant cells, mainly using a Bordetella pertussis calmodulin-dependent adenylate cyclase (Cya) reporter system (Casper-Lindley et al., 2002; Cunnac et al., 2004; Schechter et al., 2004; Sory \& Cornelis, 1994).

In R. solanacearum, the components of the Hrp TTSS are encoded within a large $h r p / h r c$ gene cluster and their expressions are controlled by an AraC-type transcriptional activator $\mathrm{HrpB}$, which is also encoded within the $h r p$ gene cluster (Genin et al., 1992; Van Gijsegem et al., 1995). Mutations in hrc genes result in the complete loss of the ability of the pathogen to proliferate in host plants (Boucher et al., 1987). This observation provides clear evidence that the Hrp-delivered effector proteins function in plant cells to enable the growth of this pathogen in host plants. In a previous study, using a transposon-based genetic screen, we identified $30 h r p B$-regulated $h p x$ ( $h r p B$-dependent expression) genes in $R$. solanacearum strain RS1000 (Mukaihara et al., 2004). Most of the $h p x$ genes were considered to encode effector proteins because they contained a PIP (plantinducible promoter) box in the promoter region and were considered to be directly regulated by $\mathrm{HrpB}$ (Mukaihara et al., 2004). In fact, the secretion of some Hpx proteins, such as $\mathrm{Hpx} 23, \mathrm{Hp} \times 24, \mathrm{Hp} \times 25$ and $\mathrm{Hp} 26$, into culture media via the Hrp TTSS has been observed (Tamura et al., 2005). In this study, adding the above $30 h p x$ genes and the wellknown HrpB-regulated popABC genes (Arlat et al., 1994; Guéneron et al., 2000), we newly cloned 11 additional candidate effector genes that share homology with known $h p x$ genes from $R$. solanacearum RS1000. Using a Cya reporter system, we investigated the translocation of these gene products into plant cells via the Hrp TTSS.

\section{METHODS}

Bacterial strains, media and culture conditions. The bacterial strains used in this study are listed in Table 1. All of the $R$. solanacearum strains are derivatives of RS1000 (race 1 biovar 4 phylotype I) (Mukaihara et al., 2004). RS1002 is a spontaneous $\mathrm{Nal}^{\mathrm{r}}$ derivative of RS1000 (Mukaihara et al., 2004). RS1085 is an EPS derivative of RS1002. In RS1085, the high production of EPSs and the formation of fluid colonies are eliminated. We used derivatives of the strain in this study because we can easily apply any genetic manipulations to the strain for comparison with the wild-type. E. coli S17-1 (Simon et al., 1983) was used as a host for oriT/mob plasmids in conjugation experiments. BG medium (Boucher et al., 1985 ) was used for the growth of $R$. solanacearum strains at $28{ }^{\circ} \mathrm{C}$ and was also used as the $h r p$-repressing medium. An $h r p$-inducing medium was prepared with the following composition: $50 \mathrm{mM}$ potassium phosphate buffer, $7.6 \mathrm{mM}\left(\mathrm{NH}_{4}\right)_{2} \mathrm{SO}_{4}, 1.7 \mathrm{mM} \mathrm{MgCl}_{2}$ and $1.7 \mathrm{mM} \mathrm{NaCl}$ supplemented with $10 \mathrm{mM}$ glutamate and $10 \mathrm{mM}$ sucrose (Tamura et al. 2005). The E. coli strains were grown at $37{ }^{\circ} \mathrm{C}$ in LB medium (Sambrook et al., 1989). Antibiotics were added to the media as described previously (Mukaihara et al., 2004).

Construction of $\boldsymbol{R}$. solanacearum mutants expressing Cya fusion protein and adenylate cyclase activity assay. For the construction of $R$. solanacearum mutants expressing the Cya fusion protein, a plasmid-based system was used as described previously (Murata et al., 2006). Briefly, a DNA fragment encoding a part (first 127-371 aa) or the full length of the protein was amplified from each candidate effector gene and inserted into pARO-HA-'Cya (Murata et al., 2006). Primer sets used for the study are listed in Supplementary Table S1 (available with the online version of this paper). The resulting plasmid was integrated into the genome of $R$. solanacearum strain RS1085 and its $\Delta h r p Y$ derivative, RS1254, by a single homologous recombination event at the native locus after conjugation with E. coli S17-1 as previously described (Mukaihara et al., 2004). The resulting strains expressing the cya fusion genes from their original promoters were used for in planta adenylate cyclase activity assay. For the in vitro adenylate cyclase activity assay, an $h r p B^{\mathrm{c}}(h r p B$ constitutive) mutant was constructed for each $R$. solanacearum strain by the electroporation of RS1239 chromosomal DNA and the selection of $\mathrm{Gm}^{\mathrm{r}}$ recombinants as described previously (Mukaihara et al., 2004). The resulting $h r p B^{c}$ mutants were used in the assay. The primers and plasmids used to construct the Cya fusions are listed in Supplementary Table S1. The adenylate cyclase activity assay and cAMP extraction protocols have been described (Murata et al., 2006).

Table 1. Bacterial strains

\begin{tabular}{|c|c|c|}
\hline Strain & Description & Reference \\
\hline \multicolumn{3}{|c|}{ R. solanacearum } \\
\hline RS1002 & RS1000 Nal ${ }^{\mathrm{r}}$ & Mukaihara et al. (2004) \\
\hline RS1085 & RS1002 $\Delta e p s P$ & Mukaihara et al. (2004) \\
\hline RS1204 & RS1085 $\Delta(h r p B-h r c T)$ & Murata et al. (2006) \\
\hline RS1239 & $\begin{array}{l}\text { RS1085 RSc2836:: mini-Tn } 5 h r p B \text { [constitutively } \\
\left.\text { expressing } h r p B\left(h r p B^{c}\right)\right]\end{array}$ & Tamura et al. (2005) \\
\hline RS1254 & RS1085 $\Delta h r p Y$ & Mukaihara et al. (2004) \\
\hline RS1312 & RS1254hrpB $B^{\mathrm{c}}$ & This study \\
\hline \multicolumn{3}{|l|}{ E. coli } \\
\hline JM109 & $\begin{array}{l}\text { recA1 supE44 endA1 hsdR17 gyrA96 relA1 } \Delta(\text { lac-proAB }) \mathrm{F}^{\prime} \\
{\left[\text { traD36 proAB }^{+} \text {lacl }^{q} \text { lacZ } \Delta \mathrm{M} 15\right]}\end{array}$ & Sambrook et al. (1989) \\
\hline S17-1 & thi pro $h s d R^{-} h s d M^{+} \operatorname{rec} A[\mathrm{chr}:: \mathrm{RP} 4-2-\mathrm{Tc}:: \mathrm{Mu}-\mathrm{Km}:: \operatorname{Tn} 7]$ & Simon et al. (1983) \\
\hline
\end{tabular}




\section{RESULTS}

\section{Cloning of candidate effector genes from $R$. solanacearum RS1000}

Based on the complete genome sequence of strain GMI1000 (Salanoubat et al., 2002), 11 additional candidate effector genes were newly cloned from strain RS1000 (Table 2). Ten of these genes are homologues of $h p x$ genes that were identified in a previous study: RSp0099, RSp0846 and RSc2139 (similar to $h p x 4$ and $h p x 10$ ); RSp0296, RSp0930, RSp1374, RSc3401 and RSc2130 (similar to $h p \times 30$ ) and RSp0028 and RSp0914 [type I/GALA leucine-rich repeat (LRR) protein genes] (Mukaihara et al., 2004). During sequencing analysis, we found a gene RSp0845 encoded downstream of RSp0846. Although RSp0845 did not show similarity to known genes found in databases, this was added to the list because a PIP box motif is present in its putative promoter region, and therefore this gene was considered to be a candidate effector gene (Table 2). Sequence analysis revealed that, in eight of the 11 candidate effector genes, a PIP box motif (consensus $5^{\prime}-\mathrm{TTCGB}^{-} \mathrm{N}_{15}$-TTCGB-3') and a probable -10 sequence (consensus $5^{\prime}$-YANNRT- $3^{\prime}$ ) are present in the putative promoter region (Table 2), suggesting that these genes are regulated by $h r p B$. No PIP box motif was observed upstream of RSc2139, RSc2130 and RSp0914.

\section{Construction and expression of Cya fusion of candidate effector proteins in $R$. solanacearum}

Our previous genetic screening resulted in the identification of $30 h r p B$-regulated genes, designated $h p x$ ( $h r p B-$ dependent expression), and three well-known $h r p B$-regulated genes, $p \circ p A, p o p B$ and $p o p C$ (DDBJ accession no. $\mathrm{AB} 302224)$, as candidate effector genes in $R$. solanacearum RS1000 (Mukaihara et al., 2004). By adding the 11 candidates newly isolated in this study (Table 2) to the above-mentioned 33 candidates, 44 genes in total were examined in this study in terms of whether the encoded protein product can be translocated into plant cells as an effector protein. Using a plasmid integration system, we constructed a series of $R$. solanacearum $\mathrm{hrp}^{+}$and Hrpdeficient $\Delta h r p Y$ strains expressing a candidate effector protein in which an adenylate cyclase domain (Cya) of $B$. pertussis adenylate cyclase toxin CyaA was C-terminally fused with a part (first 127-371 aa) or the full length of the protein. It is important to note that, in these strains, cya fusion genes are expressed from their original promoters on the chromosome of RS1000 (see Methods for the construction of Cya fusions). Then, to check the activity of the Cya fusion protein, the $h r p B^{\mathrm{C}}$ mutation was introduced into each strain. When the Cya fusion protein was expressed in the $h r p B^{c}$ background in an $h r p$-inducing medium, calmodulin-dependent adenylate cyclase activities were detected in cell lysates; these were comparable in the $h r p^{+}$and $\Delta h r p Y$ strains even though the expression levels varied among the fusion proteins (Table 3 ). This indicates that functional fusion proteins were expressed at similar levels in both series of strains.

Before the in planta Cya activity assay, to determine whether the 11 candidate effector genes newly cloned in this study are regulated by $h r p B$, we compared the expression level of the Cya fusion protein between the

Table 2. Newly cloned candidate effector genes from R. solanacearum RS1000

\begin{tabular}{|c|c|c|c|c|c|}
\hline \multirow[t]{2}{*}{ Gene } & \multirow{2}{*}{$\begin{array}{c}\text { DDBJ }^{\star} \\
\text { accession no. }\end{array}$} & \multicolumn{2}{|r|}{ Probable PIP box and -10 sequence $\dagger$} & \multirow{2}{*}{$\begin{array}{c}\text { Protein } \\
\text { size }(a a)\end{array}$} & \multirow[t]{2}{*}{ Similar Hpx protein } \\
\hline & & Position & Nucleotide sequence & & \\
\hline RSp0846 & AB302226 & -98 & TTCGG- $\mathrm{N}_{15}$-TTCGG-N ${ }_{28}$-CACATT & 1238 & $\begin{array}{c}\text { Hpx4 }(53.7 \% \text { identity, } 80.7 \% \text { similarity }) \text { and } \\
\text { Hpx10 }(22.2 \% \text { identity, } 63.8 \% \text { similarity })\end{array}$ \\
\hline RSc2139 & AB302227 & - & Not found & 1067 & $\begin{array}{c}\text { Hpx4 }(20.4 \% \text { identity, } 63.1 \% \text { similarity }) \text { and } \\
\text { Hpx10 (22.0\% identity, } 65.7 \% \text { similarity })\end{array}$ \\
\hline RSp0296 & AB302230 & -421 & TTCGC-N 15 -TTCGC-N ${ }_{33}$-TACACT & 2338 & Hрx30 (38.0\% identity, $73.5 \%$ similarity) \\
\hline RSp0930 & AB302231 & -470 & TGCGC-N 15 -TTCGC-N 33 -TATCGT & 2208 & Hpx30 (24.8 \% identity, $66.0 \%$ similarity) \\
\hline RSp1374 & AB302232 & -303 & TTCGG-N ${ }_{15}$-TTCGT-N 31 -CATTCT & 2504 & Hрx30 (48.4\% identity, $82.3 \%$ similarity) \\
\hline RSc3401 & AB302233 & -683 & TTCGT-N 15 -TTCGC-N ${ }_{31}$-TACACT & 2353 & Hрx30 (22.2 \% identity, $65.4 \%$ similarity) \\
\hline RSp0914 & AB302229 & - & Not found & 493 & Type I/GALA LRR protein \\
\hline
\end{tabular}

${ }^{\star}$ DDBJ, DNA Data Bank of Japan.

$\nmid$ Position indicates position of the first nucleotide (T) of the first TTCGB motif of a PIP box (consensus $5^{\prime}$-TTCGB-N $15^{-}{ }^{-T T C G B-3}{ }^{\prime}$ ) relative to the start codon of an ORF. Conserved nucleotides of a PIP box are shown in bold. N means the number of spacer nucleotides between the first and second TTCGB motifs of a PIP box or between the second motif of a PIP box and a -10 sequence (consensus $5^{\prime}$-YANNRT- $3^{\prime}$ ). 
Table 3. In vitro adenylate cyclase activity of cell lysates from $R$. solanacearum $h r p^{+}$and $\Delta h r p Y$ strains expressing the Cya fusion protein in the $h r p B^{\mathrm{c}}$ background

\begin{tabular}{|c|c|c|c|c|}
\hline \multirow[t]{3}{*}{ Fusion protein ${ }^{\star}$} & \multicolumn{4}{|c|}{ cAMP level $\dagger$ (fold increase $\ddagger$ ) } \\
\hline & \multicolumn{2}{|c|}{ + Calmodulin } & \multicolumn{2}{|c|}{-Calmodulin } \\
\hline & $h r p B^{c}$ & $h r p B^{c} \Delta h r p Y$ & $h r p B^{c}$ & $h r p B^{c} \Delta h r p Y$ \\
\hline None & $0.01 \pm 0.002(1.0)$ & $0.01 \pm 0.002$ & $0.01 \pm 0.002$ & $0.01 \pm 0.002(1.0)$ \\
\hline Hpx1' -'Cya & $8.53 \pm 0.67(853)$ & $9.22 \pm 0.45(920)$ & $0.04 \pm 0.01(4.0)$ & $0.06 \pm 0.01(7.0)$ \\
\hline Hpx2'-'Cya & $46.2 \pm 3.55(4620)$ & $45.3 \pm 5.29(4530)$ & $0.15 \pm 0.03(15.0)$ & $0.40 \pm 0.03(40.0)$ \\
\hline Нpx3'-'Сya & $2.26 \pm 0.37(226)$ & $2.92 \pm 0.08(292)$ & $0.01 \pm 0.002$ & $0.01 \pm 0.001$ \\
\hline $\mathrm{Hpx}_{1-230}{ }^{\prime}-^{\prime} \mathrm{Cya}$ & $3.66 \pm 0.49(366)$ & $11.6 \pm 0.72(1160)$ & $0.02 \pm 0.004(2.0)$ & $0.03 \pm 0.01$ \\
\hline Hpx5 (LrpE) ${ }_{1-230}$-' $^{\prime}$ Cya & $30.0 \pm 2.52(3000)$ & $28.9 \pm 2.40(2890)$ & $0.12 \pm 0.02(12.0)$ & $0.07 \pm 0.01$ \\
\hline Hpx6' -'Cya & $1427 \pm 154(142700)$ & $1474 \pm 140(147400)$ & $1.15 \pm 0.11(115)$ & $1.09 \pm 0.11(109)$ \\
\hline Hpx7' -'Cya & $228 \pm 12.5(22800)$ & $223 \pm 17.1(22300)$ & $0.28 \pm 0.02(28.0)$ & $0.58 \pm 0.06(58.0)$ \\
\hline Нpx $8^{\prime}-{ }^{\prime}$ Сya & $26.1 \pm 2.38(2610)$ & $16.4 \pm 1.17(1640)$ & $0.04 \pm 0.01(4.0)$ & $0.03 \pm 0.003(3.0)$ \\
\hline Нpx $9_{1-230}{ }^{\prime}-{ }^{\prime}$ Саa & $4.47 \pm 0.50(447)$ & $14.8 \pm 0.25(1480)$ & $0.02 \pm 0.003$ & $0.07 \pm 0.01(7.0)$ \\
\hline Hpx $10_{1-230}{ }^{\prime}{ }^{\prime}$ Сya & $139 \pm 23.0(13900)$ & $306 \pm 31.6(30600)$ & $0.23 \pm 0.04$ & $0.38 \pm 0.01(38.0)$ \\
\hline Hpx $11_{1-230}{ }^{\prime}-$ Cya & $9.34 \pm 0.93(934)$ & $10.4 \pm 1.24(1040)$ & $0.03 \pm 0.01(3.0)$ & $0.02 \pm 0.003$ \\
\hline Hpx12'-'Cya & $300 \pm 14.4(30000)$ & $385 \pm 13.7(38500)$ & $0.27 \pm 0.01(27.0)$ & $0.51 \pm 0.03(51.0)$ \\
\hline Hpx13 (GALA6) ${ }_{1-190}{ }^{\prime}-{ }^{\prime}$ Cya & $18.1 \pm 4.33(1810)$ & $36.2 \pm 3.42(3620)$ & $0.10 \pm 0.03(10.0)$ & $0.13 \pm 0.02(13.0)$ \\
\hline Hpx14 (GALA7) ${ }_{1-229}$-'Cya $^{\prime}$ & $194 \pm 21.6(19400)$ & $176 \pm 31.0(17600)$ & $0.54 \pm 0.08(54.0)$ & $0.52 \pm 0.05(52.0)$ \\
\hline Hpx15 (GALA4) ${ }_{1-168}$-' $^{\prime}$ Cya & $31.3 \pm 3.13(3130)$ & $43.1 \pm 1.70(4310)$ & $0.12 \pm 0.02(12.0)$ & $0.12 \pm 0.02(12.0)$ \\
\hline Hpx16 (GALA5) ${ }_{1-170}$-' $^{\prime}$ Cya & $3.54 \pm 0.65(354)$ & $4.80 \pm 0.51(480)$ & $0.02 \pm 0.002$ & $0.02 \pm 0.004(2.0)$ \\
\hline Hpx $17_{1-230}{ }^{\prime}-^{\prime}$ Cya & $26.3 \pm 2.08(2630)$ & $35.7 \pm 1.53(3570)$ & $0.12 \pm 0.01(12.0)$ & $0.40 \pm 0.06(40.0)$ \\
\hline Hpx18'-'Сya & $449 \pm 54.9(44900)$ & $645 \pm 32.6(64500)$ & $0.58 \pm 0.07(58.0)$ & $0.64 \pm 0.05(64.0)$ \\
\hline Hpx19' -'Cya & $3351 \pm 317(335100)$ & $3620 \pm 251(362000)$ & $0.62 \pm 0.04(62.0)$ & $0.71 \pm 0.09(71.0)$ \\
\hline Hpx20 (GALA2) ${ }_{1-127^{\prime}-{ }^{\prime} \text { Cya }}$ & $14.1 \pm 1.09$ & $17.6 \pm 2.53(1760)$ & $0.07 \pm 0.01(7.0)$ & $0.15 \pm 0.02(15.0)$ \\
\hline Hpx $21_{1-230}{ }^{\prime}{ }^{\prime}$ Cya & $16.3 \pm 2.75(1630)$ & $27.1 \pm 1.92(2710)$ & $0.06 \pm 0.01(6.0)$ & $0.12 \pm 0.01(12.0)$ \\
\hline Нpx $22_{1-230}{ }^{\prime}-$ Cya & $0.23 \pm 0.02(23.0)$ & $0.25 \pm 0.02(25.0)$ & $0.01 \pm 0.001$ & $0.01 \pm 0.002(1.0)$ \\
\hline Нpx23'-'Сya & $19.2 \pm 2.40(1920)$ & $22.3 \pm 1.62(2230)$ & $0.09 \pm 0.01(9.0)$ & $0.11 \pm 0.02(11.0)$ \\
\hline Нpx $24_{1-230}{ }^{\prime}-$ Cya & $87.9 \pm 4.91(8790)$ & $159 \pm 17.3(15900)$ & $0.14 \pm 0.01(14.0)$ & $0.20 \pm 0.02(20.0)$ \\
\hline Нpx $25_{1-230}{ }^{\prime}-$ Сya & $260 \pm 18.3(26000)$ & $312 \pm 33.2(31200)$ & $0.45 \pm 0.03(45.0)$ & $0.81 \pm 0.05(81.0)$ \\
\hline Нpx $26_{1-230}{ }^{\prime}-^{\prime}$ Cya & $134 \pm 12.7(13400)$ & $229 \pm 16.3(22900)$ & $0.06 \pm 0.01(6.0)$ & $0.16 \pm 0.01$ \\
\hline Нpx $27_{1-230}{ }^{\prime}-{ }^{\prime}$ Суa & $5.48 \pm 0.91(548)$ & $9.31 \pm 0.35(931)$ & $0.04 \pm 0.01$ & $0.03 \pm 0.01$ \\
\hline Нpx $28_{1-230}{ }^{\prime}-$ Суa & $10.5 \pm 0.92(1410)$ & $17.6 \pm 1.73(1760)$ & $0.08 \pm 0.01(8.0)$ & $0.11 \pm 0.01(11.0)$ \\
\hline Нpx29 ${ }_{1-230}{ }^{\prime}-$ Суа & $0.67 \pm 0.03(67.0)$ & $0.82 \pm 0.12(82.0)$ & $0.01 \pm 0.002(1.0)$ & $0.01 \pm 0.001$ \\
\hline Нpx30 $1-230^{\prime}-^{\prime}$ Суа & $1.03 \pm 0.09(103)$ & $1.26 \pm 0.08(126)$ & $0.01 \pm 0.001(1.0)$ & $0.01 \pm 0.001$ \\
\hline PopA' -'Cya & $4119 \pm 62.9(411900)$ & $3815 \pm 231(381500)$ & $3.79 \pm 0.28(379)$ & $4.25 \pm 0.22(425)$ \\
\hline 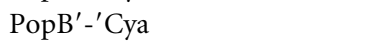 & $1870 \pm 178(187000)$ & $2586 \pm 173(258600)$ & $1.10 \pm 0.08(110)$ & $1.45 \pm 0.12(145)$ \\
\hline PopC $_{1-230}$-'Cya $^{\prime}$ & $8.01 \pm 1.62(801)$ & $9.50 \pm 1.19(950)$ & $0.02 \pm 0.002(2.0)$ & $0.03 \pm 0.002$ \\
\hline RSp0099 (Нpx31) $1-230^{\prime}$-'Cya & $12.1 \pm 0.62(1210)$ & $21.7 \pm 0.95(2170)$ & $0.12 \pm 0.01(12.0)$ & $0.07 \pm 0.001$ \\
\hline RSp0846 (Нpx32) ${ }_{1-230}{ }^{\prime}-{ }^{\prime}$ Cya & $0.33 \pm 0.03(33.0)$ & $0.36 \pm 0.02(36.0)$ & $0.01 \pm 0.001$ & $0.01 \pm 0.001$ \\
\hline RSp0845 (Hpx33) ${ }_{1-230}{ }^{\prime}$-'Cya $^{-}$ & $633 \pm 20.5(63300)$ & $807 \pm 30.6(80700)$ & $0.11 \pm 0.01(11.0)$ & $0.32 \pm 0.03(32.0)$ \\
\hline RSc21391-230' -'Cya & $0.16 \pm 0.01(16.0)$ & $0.18 \pm 0.01(18.0)$ & $0.01 \pm 0.001$ & $0.01 \pm 0.002$ \\
\hline RSp0296 (Нpx34) $)_{1-233^{\prime}-{ }^{\prime} \text { Cya }}$ & $8.96 \pm 1.46(896)$ & $12.1 \pm 0.40(1210)$ & $0.04 \pm 0.01(4.0)$ & $0.05 \pm 0.01(5.0)$ \\
\hline RSp0930 (Нpx35) ${ }_{1-255^{\prime}-{ }^{\prime} \text { Cya }}$ & $0.32 \pm 0.06(32.0)$ & $0.25 \pm 0.02(25.0)$ & $0.01 \pm 0.001$ & $0.01 \pm 0.001$ \\
\hline RSp1374 (Нpx36) ${ }_{1-371}{ }^{\prime}-{ }^{\prime}$ Cya & $0.67 \pm 0.05(67.0)$ & $0.76 \pm 0.04(76.0)$ & $0.01 \pm 0.001$ & $0.01 \pm 0.001$ \\
\hline RSc3401 (Нpx37) $)_{1-236}$-'Cya $^{\prime}$ & $1.00 \pm 0.07(100)$ & $0.86 \pm 0.24(86.0)$ & $0.01 \pm 0.001(1.0)$ & $0.01 \pm 0.001$ \\
\hline $\mathrm{RSc} 2130_{1-222^{\prime}-{ }^{\prime} \mathrm{Cya}}$ & $0.15 \pm 0.01(15.0)$ & $0.08 \pm 0.01(8.0)$ & $0.01 \pm 0.001$ & $0.01 \pm 0.001$ \\
\hline RSp0028 (GALA3) ${ }_{1-220}{ }^{\prime}-{ }^{\prime}$ Cya & $0.98 \pm 0.04(98.0)$ & $1.05 \pm 0.13(105)$ & $0.01 \pm 0.004$ & $0.01 \pm 0.002(1.0)$ \\
\hline RSp0914 (GALA1) $1-165{ }^{\prime}$-'Cya & $0.20 \pm 0.03(20.0)$ & $0.25 \pm 0.06(25.0)$ & $0.01 \pm 0.003(1.0)$ & $0.01 \pm 0.005$ \\
\hline
\end{tabular}

*Subscript number indicates the position of amino acids of proteins that are fused with the Cya reporter protein. Number is not shown in candidate effector proteins of which the full length of the protein is fused with the Cya reporter.

$\dagger$ Adenylate cyclase activity ( $\mathrm{nmol}$ cAMP per mg total soluble protein) was measured as described in Methods; the mean \pm SD of values from three independent experiments is shown.

$\ddagger$ Fold increase indicates the ratio of activity of a strain expressing the Cya fusion protein to that of the parental strain that does not express Cya. 
$h r p B^{c}$ and $\triangle h r p B$ strains. The Cya activities of eight genes, which have a PIP box motif in the putative promoter region (Table 2), increased 8-1200-fold in the $h r p B^{c}$ background compared with the $\triangle h r p B$ background (Table 4). This indicates that the expression of the PIP-boxcontaining genes is indeed regulated by $h r p B$. Hence, we renamed these genes $h p x$ ( $h r p B$-regulated expression) as follows: $h p \times 31$ (RSp0099), $h p \times 32$ (RSp0846), $h p \times 33$ (RSp0845), $h p x 34$ (RSp0296), $h p x 35$ (RSp0930), $h p \times 36$ (RSp1374), $h p \times 37$ (RSc3401). In the case of the remaining three genes, which have no PIP box motif (RSc2139, RSc2130 and RSp0914) (Table 2), the Cya activities were similar in both $h r p B^{c}$ and $\triangle h r p B$ backgrounds but their expression levels were quite low (Table 4). It was therefore considered that these genes are not efficiently expressed in the artificial $h r p$-inducing medium used in this study.

\section{Identification of novel $R$. solanacearum type III effector proteins}

R. solanacearum $h r p^{+}$and $\Delta h r p Y$ strains expressing the Cyafused candidate effector protein in the $h r p B^{+}$background were inoculated into host eggplant leaves, and the cAMP levels of the inoculated leaves were measured $12 \mathrm{~h}$ after inoculation (Table 5). When the $h r p^{+}$strains were used, in 34 of the 44 Cya fusions the cAMP level of inoculated leaves increased 65-9300-fold compared with the background level (Table 4). In all the positive fusions, such an increase in the cAMP level was not observed when the $\Delta h r p Y$ strains were used (Table 5), whereas the fusion proteins were expressed at similar levels in both strains (Table 3 ). This clearly indicates that these 34 proteins are effector proteins translocated into plant cells via the Hrp TTSS.

\section{DISCUSSION}

In this study, we identified 34 type III effector proteins from $R$. solanacearum RS1000 using a Cya reporter system (Table 5). We identified three effector families composed of more than four members, namely, the Hpx4, Hpx30 and GALA families. The Hpx4 family in R. solanacearum RS1000 consists of the Hpx4, Hpx10, Нpx31 and Hpx32 effectors. The RSc2139 gene also encoded a protein belonging to the Hpx4 family (Table 2) but was considered to be nonfunctional because we could not detect the translocation of its product into plant cells by in planta Cya activity assay (Table 5). One of the Hpx4 family proteins, Hpx31, has been reported to be translocated into plant cells via the Hrp TTSS and is designated RipA (Ralstonia effector injected into plant cells) in R. solanacearum GMI1000 (Cunnac et al., 2004). We herein demonstrated that $\mathrm{Hpx} 31$ and the other three members of this family are translocated into plant cells in RS1000 (Table 5). The Hpx4 family proteins do not contain any known protein motifs found in databases (Table 2), and therefore it is difficult to predict their functions in plant cells. However, considering both their amplified copy number in $R$. solanacearum and the fact that similar proteins are distributed in other phytopathogenic bacteria, such as Xanthomonas spp. and Acidovorax avene subsp. citrulli (data not shown), the Hpx4 effector family might have important functions in pathogenicity.

The Hpx30 family is a novel $R$. solanacearum effector family. The five genes encoding this type of protein, namely RSc1839 (Hpx30), RSp0296 (Hpx34), RSp0930 (Hpx35), RSp1374 (Hpx36) and RSc3401 (Hpx37), have not been detected by the genome-wide screens for $h r p B$-regulated candidate effector genes in strain GMI1000 (Cunnac et al.,

Table 4. In vitro adenylate cyclase activity of cell lysates from $R$. solanacearum $h r p B^{\mathrm{C}}$ and $\Delta h r p B$ strains expressing Cya fusions of newly cloned candidate effectors

\begin{tabular}{|c|c|c|c|c|}
\hline \multirow[t]{2}{*}{ Fusion protein ${ }^{\star}$} & \multicolumn{2}{|c|}{ cAMP level $\dagger$} & \multirow[t]{2}{*}{ Ratio of $h r p B^{c} / \Delta h r p B$} & \multirow{2}{*}{$\begin{array}{l}\text { HrpB-dependent } \\
\text { expression }\end{array}$} \\
\hline & $h r p B^{c}$ & $\Delta h r p B$ & & \\
\hline RSp0099 (Нpx31) ${ }_{1-230}$-' $^{\prime}$ Сya & $12.1 \pm 0.62$ & $0.17 \pm 0.01$ & 71.2 & Yes \\
\hline RSp0846 (Нpx32) ${ }_{1-230^{\prime}-{ }^{\prime} \text { Cya }}$ & $0.33 \pm 0.03$ & $0.01 \pm 0.004$ & 33.0 & Yes \\
\hline RSp0296 (Нpx34) ${ }_{1-233^{\prime}-{ }^{\prime} \text { Cya }}$ & $8.96 \pm 1.46$ & $0.08 \pm 0.01$ & 112 & Yes \\
\hline RSp0930 (Нpx35) ${ }_{1-255^{\prime}-{ }^{\prime} \text { Cya }}$ & $0.32 \pm 0.06$ & $0.01 \pm 0.001$ & 32.0 & Yes \\
\hline RSp1374 (Нpx36) ${ }_{1-371}{ }^{\prime}-{ }^{\prime}$ Cya & $0.67 \pm 0.05$ & $0.01 \pm 0.004$ & 67.0 & Yes \\
\hline RSc3401 (Нpx37) ${ }_{1-236}$-' $^{\prime}$ Cya & $1.00 \pm 0.07$ & $0.09 \pm 0.01$ & 11.1 & Yes \\
\hline RSc2130 ${ }_{1-222}{ }^{\prime}-{ }^{\prime}$ Cya & $0.15 \pm 0.01$ & $0.08 \pm 0.01$ & 1.8 & No \\
\hline RSp0028 (GALA3) $)_{1-220}{ }^{\prime}-^{\prime}$ Cya & $0.98 \pm 0.05$ & $0.12 \pm 0.02$ & 8.2 & Yes \\
\hline
\end{tabular}

*Subscript number indicates the position of amino acids of proteins that are fused with the Cya reporter protein. Number is not shown in candidate effector proteins in which the full length of the protein is fused with the Cya reporter.

$\dagger$ Adenylate cyclase activity ( $\mathrm{nmol}$ cAMP per mg total soluble protein) was measured as described in Methods; the mean \pm SD of values from three independent experiments is shown. 
Table 5. cAMP level of plant leaves inoculated with $R$. solanacearum $h r p^{+}$and $\Delta h r p Y$ strains expressing the Cya fusion protein in the wild-type $h r p B$ background

\begin{tabular}{|c|c|c|c|c|}
\hline \multirow[t]{2}{*}{ Fusion protein ${ }^{\star}$} & \multicolumn{2}{|c|}{ cAMP level $\dagger$ (fold increase $\ddagger$ ) } & \multirow{2}{*}{$\begin{array}{c}\text { Ratio of } h r p^{+} / \\
\Delta h r p Y\end{array}$} & \multirow{2}{*}{$\begin{array}{l}\text { Hrp-dependent } \\
\text { translocation into } \\
\text { plant cells }\end{array}$} \\
\hline & $h r p^{+}$ & $\Delta h r p Y$ & & \\
\hline None & $0.02 \pm 0.004(1.0)$ & $0.02 \pm 0.004(1.0)$ & 1.0 & - \\
\hline Hpx1'-'Cya & $0.06 \pm 0.01(3.0)$ & $0.07 \pm 0.01$ & 0.9 & No \\
\hline Нpx2'-'Сya & $75.3 \pm 3.81(3765)$ & $0.02 \pm 0.002(1.0)$ & 3765 & Yes \\
\hline Нpx3'-'Суа & $0.02 \pm 0.002(1.0)$ & $0.01 \pm 0.002(0.5)$ & 2.0 & No \\
\hline Hpx $4_{1-230}{ }^{\prime}-{ }^{\prime}$ ya & $25.7 \pm 2.98(1285)$ & $0.01 \pm 0.002(0.5)$ & 2570 & Yes \\
\hline Hpx5 (LrpE) ${ }_{1-230}$-' $^{\prime}$ Cya & $0.04 \pm 0.01(2.0)$ & $0.04 \pm 0.004$ & 1.0 & No \\
\hline Hpx6' -'Сya & $0.04 \pm 0.01(2.0)$ & $0.02 \pm 0.002$ & 2.0 & No \\
\hline Hpx7' -'Сya & $0.10 \pm 0.01(5.0)$ & $0.12 \pm 0.01(6.0)$ & 0.8 & No \\
\hline Нpx8' -'Сya & $18.3 \pm 1.87(915)$ & $0.02 \pm 0.003(1.0)$ & 915 & Yes \\
\hline Нpx $9_{1-230}{ }^{\prime}-\mathrm{Cya}$ & $59.5 \pm 2.40(2975)$ & $0.01 \pm 0.005(0.5)$ & 5950 & Yes \\
\hline Нpx $10_{1-230}{ }^{\prime}{ }^{\prime}$ Cya & $57.6 \pm 4.29(2880)$ & $0.04 \pm 0.001$ & 1440 & Yes \\
\hline Hpx $11_{1-230}{ }^{\prime}-^{\prime}$ Cya & $83.0 \pm 14.9(4150)$ & $0.06 \pm 0.01$ & 1383 & Yes \\
\hline Hpx12'-'Сya & $0.09 \pm 0.01$ & $0.03 \pm 0.01$ & 3.0 & No \\
\hline Hpx13 (GALA6) ${ }_{1-190}$-' Cya $^{-}$ & $54.0 \pm 7.43(2700)$ & $0.03 \pm 0.01$ & 1800 & Yes \\
\hline Hpx14 (GALA7) ${ }_{1-229^{\prime}-{ }^{\prime} \text { Cya }}$ & $187 \pm 15.1(9350)$ & $0.02 \pm 0.002(1.0)$ & 9350 & Yes \\
\hline Hpx15 (GALA4) ${ }_{1-168}{ }^{\prime}-{ }^{\prime}$ Cya & $27.5 \pm 5.44(1375)$ & $0.02 \pm 0.002(1.0)$ & 1375 & Yes \\
\hline Hpx16 (GALA5) ${ }_{1-170}$-' $^{\prime}$ Cya & $4.53 \pm 0.76(227)$ & $0.03 \pm 0.003$ & 151 & Yes \\
\hline $\mathrm{Hpx}_{17-230^{\prime}-{ }^{\prime} \mathrm{Cya}}$ & $11.3 \pm 3.61(565)$ & $0.07 \pm 0.02$ & 161 & Yes \\
\hline Hpx18'-'Cya & $75.7 \pm 18.2(3785)$ & $0.03 \pm 0.01$ & 2523 & Yes \\
\hline Hpx19'-'Сya & $0.05 \pm 0.01(2.5)$ & $0.05 \pm 0.01(2.5)$ & 1.0 & No \\
\hline Hpx20 (GALA2) ${ }_{1-127^{\prime}-{ }^{\prime} \text { Cya }}$ & $89.5 \pm 8.32(4475)$ & $0.02 \pm 0.002$ & 4475 & Yes \\
\hline Нpx $21_{1-230}{ }^{\prime}-$ Суa & $14.6 \pm 2.78(730)$ & $0.02 \pm 0.01$ & 730 & Yes \\
\hline Hpx $22_{1-230}{ }^{\prime}-\mathrm{Cya}$ & $0.04 \pm 0.01(2.0)$ & $0.04 \pm 0.01(2.0)$ & 1.0 & No \\
\hline Нpx23'-'Суа & $97.2 \pm 19.2(4860)$ & $0.02 \pm 0.003$ & 4860 & Yes \\
\hline Нpx $24_{1-230}{ }^{\prime}-$ Cya & $4.24 \pm 1.11(212)$ & $0.03 \pm 0.01$ & 141 & Yes \\
\hline Нpx $25_{1-230}{ }^{\prime}{ }^{\prime}$ Сya & $144 \pm 21.3(7200)$ & $0.04 \pm 0.01$ & 3600 & Yes \\
\hline Нpx $26_{1-230}{ }^{\prime}-{ }^{\prime}$ Сya & $79.2 \pm 18.9(3960)$ & $0.03 \pm 0.01$ & 2640 & Yes \\
\hline Нpx $27_{1-230}{ }^{\prime}-{ }^{\prime}$ Сya & $75.2 \pm 5.54(3760)$ & $0.02 \pm 0.01$ & 3760 & Yes \\
\hline Нpx $28_{1-230}{ }^{\prime}-{ }^{\prime}$ Суa & $71.5 \pm 4.90(3575)$ & $0.02 \pm 0.003$ & 3575 & Yes \\
\hline Нpx29 ${ }_{1-230}{ }^{\prime}-$ Суa & $98.1 \pm 8.37(4905)$ & $0.02 \pm 0.004$ & 4905 & Yes \\
\hline Нpx $30_{1-230}{ }^{\prime}-{ }^{\prime}$ Cya & $40.7 \pm 5.72(2035)$ & $0.01 \pm 0.002(0.5)$ & 4070 & Yes \\
\hline PopA'-'Cya & $49.4 \pm 5.20(2470)$ & $0.25 \pm 0.03(12.5)$ & 198 & Yes \\
\hline PopB'-'Cya & $46.9 \pm 7.08(2345)$ & $0.16 \pm 0.04$ & 293 & Yes \\
\hline PopC $_{1-230}{ }^{\prime}-{ }^{\prime}$ Cya & $122 \pm 13.7(6100)$ & $0.06 \pm 0.01$ & 2033 & Yes \\
\hline Hpx31 (RSp0099) $)_{1-230}$-'Cya $^{\prime}$ & $24.5 \pm 10.2(1225)$ & $0.03 \pm 0.002$ & 817 & Yes \\
\hline Hpx32 (RSp0846) ${ }_{1-230}$ - 'Cya $^{\prime}$ & $13.6 \pm 2.30(680)$ & $0.04 \pm 0.01$ & 340 & Yes \\
\hline Hpx33 (RSp0845) ${ }_{1-230}{ }^{\prime}-^{\prime}$ Сya & $21.9 \pm 3.79(1095)$ & $0.05 \pm 0.02(2.5)$ & 438 & Yes \\
\hline RSc2139 ${ }_{1-230^{\prime}}-{ }^{\prime}$ Cya & $0.08 \pm 0.01(4.0)$ & $0.04 \pm 0.01(2.0)$ & 2.0 & No \\
\hline Hpx34 (RSp0296) ${ }_{1-233^{\prime}-{ }^{\prime} \text { Cya }}$ & $13.7 \pm 2.67(685)$ & $0.04 \pm 0.01(2.0)$ & 343 & Yes \\
\hline Hpx35 (RSp0930) ${ }_{1-255}$-'Cya & $16.2 \pm 1.42(810)$ & $0.03 \pm 0.01(1.5)$ & 540 & Yes \\
\hline Hpx36 (RSp1374) $)_{1-371}{ }^{\prime}$-'Cya $^{-}$ & $10.8 \pm 1.18(540)$ & $0.05 \pm 0.01(2.5)$ & 216 & Yes \\
\hline Hpx37 (RSc3401) $)_{1-236}$-'Cya $^{\prime}$ & $3.27 \pm 1.20(164)$ & $0.05 \pm 0.01(2.5)$ & 65.4 & Yes \\
\hline RSc2130 $1-222^{\prime}-{ }^{\prime}$ Cya & $0.06 \pm 0.01(3.0)$ & $0.05 \pm 0.01(2.5)$ & 1.2 & No \\
\hline RSp0028 (GALA3) $)_{1-220}{ }^{\prime}-{ }^{\prime}$ Cya & $71.5 \pm 8.70(3575)$ & $0.03 \pm 0.002(1.5)$ & 2383 & Yes \\
\hline RSp0914 (GALA1) $1-165{ }^{\prime}$-'Cya & $4.23 \pm 1.50(212)$ & $0.02 \pm 0.01$ & 212 & Yes \\
\hline
\end{tabular}

${ }^{\star}$ Subscript number indicates the position of amino acids of proteins that are fused with the Cya reporter protein. Number is not shown in candidate effector proteins in which the full length of the protein is fused with the Cya reporter.

$\dagger$ Adenylate cyclase activity ( $\mathrm{nmol}$ cAMP per mg total soluble protein) was measured as described in Methods; the mean \pm SD of values from three independent experiments is shown.

$\ddagger$ Fold increase indicates the ratio of activity of a strain expressing the Cya fusion protein to that of the parental strain that does not express Cya. 
2004; Occhialini et al., 2005). This might be due to the low expression level of the $h p \times 30$ family genes in an $h r p-$ inducing medium (Mukaihara et al., 2004). The Hpx30 family effectors are 2200-2500 aa in size and appear to be the largest class of effector proteins among those identified before from animal and plant-pathogenic bacteria (Fig. 1a). The RSc2130 gene also encoded the Hpx30 family protein (Table 2) but was considered to be non-functional from the in planta Cya activity assay (Table 5). Members of this family contain a novel amino acid repeat domain, designated here as SKWP repeats, from its conserved motif (Fig. 1a). Hpx30, for instance, contains 18 tandem SKWP repeats of a 42 aa motif (Fig. 1b). Other Hpx30 family members contain 12-15 SKWP repeats (Fig. 1a). The size and the consensus amino acid residues of the motif are well conserved among the family members (Fig. 1c). As an exception, Hpx36 contains SKWP repeats of a 45 aa motif (Fig. 1c). Long and repetitive amino acid sequences have been reported to form an interaction domain for a certain ligand. The Hpx30 family proteins might interact with plant factor(s) via the SKWP repeat domain to exert virulence functions.

The third family consists of the Hpx13/GALA6, Hpx14/ GALA7, Hpx15/GALA4, Hpx16/GALA5, Hpx20/GALA2,
RSp0028/GALA3 and RSp0914/GALA1 effectors (Fig. 2a), some of which were previously called GALA or type I LRR proteins (Cunnac et al., 2004; Mukaihara et al., 2004). Although two of the GALA proteins, namely GALA6/RipG (Hpx13 in RS1000) and GALA7 (Hpx14 in RS1000) have been reported in terms of their translocation into plant cells via the Hrp TTSS (Angot et al., 2006; Cunnac et al., 2004), in this study we demonstrated that the other members from RS1000 are also translocated into plant cells (Table 5). Members of the GALA family contain large LRRs that share almost two-thirds of the entire amino acids of the protein (Fig. 2a), which comprise a new kind of bacterial LRR protein (Cunnac et al., 2004; Kajava et al., 2008; Mukaihara et al., 2004). Hpx13/GALA6 of RS1000, for instance, contains 17 tandem repeats of the LRR motif with 16 perfect LRRs of 24 aa and one imperfect LRR of 23 aa (Fig. 2b). On the other hand, the N-terminal sequence of the GALA effectors contains F-box motif-like sequences (Fig. 2a and b). Hence, these proteins resemble the plant F-box protein, a subunit of the SCF (for Skp1Cullin-F-box) complex that functions as an E3 class ubiquitin ligase (Bai et al., 1996). Indeed, Angot et al. (2006) revealed that several GALA proteins specifically interact with subsets of Arabidopsis Skp1-like (ASK) (a)

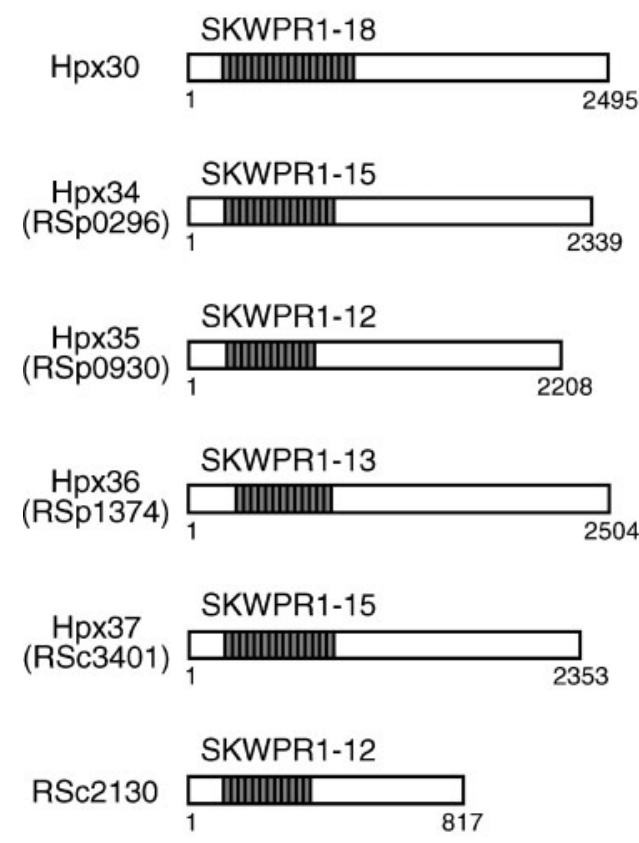

(b)

(c)

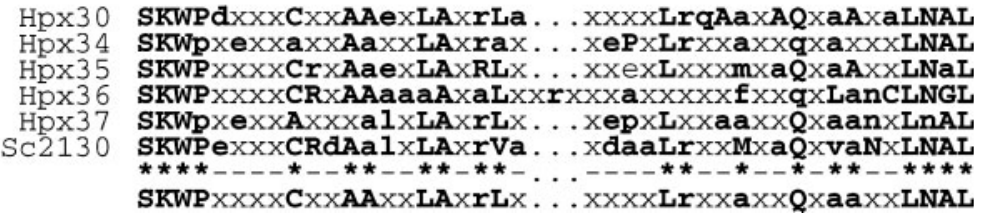

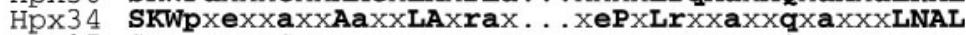
Hpx35 SKWP XXXXCrXAaexLAXRLX... XXexLXXXmXaQXaAXXLNaL SKWPXXXXCRXAAaaaAXaLXX $\times$ XXa $\times X \times X \times 1 \times \times 9 \times L a n C L N G I$ SKWpXeXXAXXXalXLAxrLX... XepXLXXaaxX0XaanXLnAI SKWPexxxCRdAal $x$ LAxrVa... xdaaLrXXMXaQxvaNxLNAL SKWP $x \times x \times C \times x A A \times x L A \times r L \times \ldots \times x \times x L r \times x a \times x Q \times a a \times x L N A L$

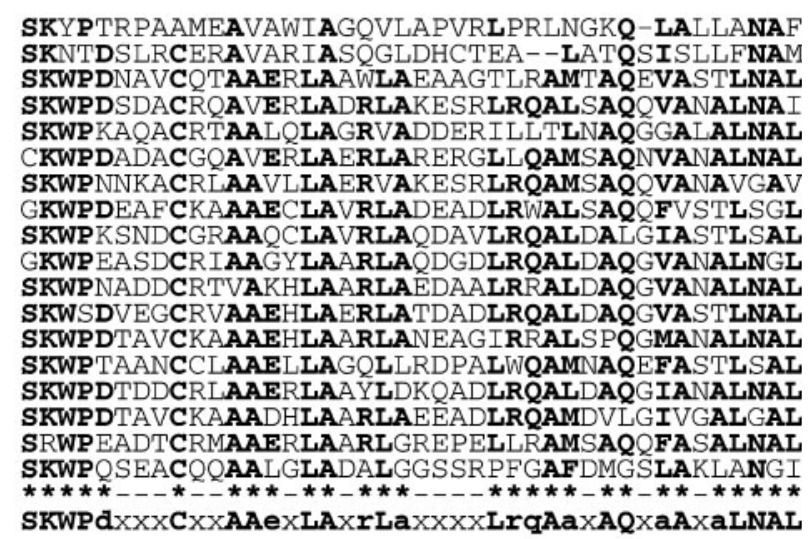


(a)

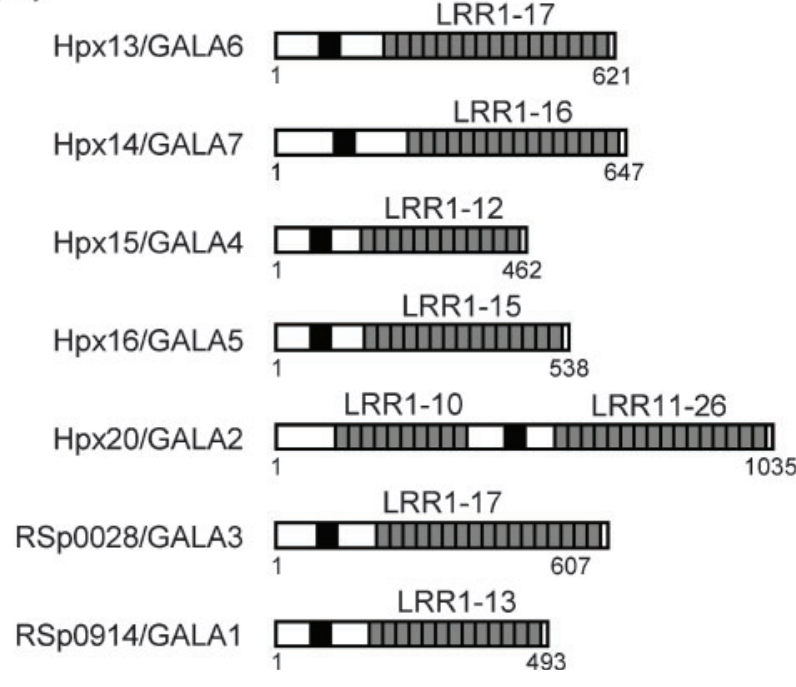

(b) MVSRIPTGNRGQGNGIEQSASSHA
TEATPWLGVNLPYGHWLPSSARSV
VSRASSLLGGLANFRWGGSASAR
PAPNIRSAAIAPAQQTLPPELWQQ
IATLAGARPRRAMREVSLELRNAS
RAVVTHLTISDPAMFRQLSLYPAI
KSVRFKGATLEALKALPPLEHL
EIGRCTGSAISAEGLAHLAAMP.
LKSLNLNGIEIGVEGARTLATSKS
LVSLSLIGCGIGDRAAQALAASR
IRSLDLSVNMIGCDGAQALADAP.
LVSLNLYSNEIGDDGARAIATSQT
LTSLELRRNGIGNAGAGAFANNTV
LRKLNLANNMIDKRGARVLAGNTS
LTELDLGGNRLGDKGARALAGNRS
LLSLKVDHNEISDEGVALAOAT
LRSLDLSFNFVGQGAGALGGNTR
LSELNLSLCGINSYSASALARNKS
LASLYLNGNRIGDDGARALAKNST
LTLLDLSRNNIQNAGAEALGGNQA
LISLKLAGNGIDDDGAAALARHPR
LTTLDLSONRIGSEGARHLAQSAT
LAELDLSENRIGPEGAKALSLSTV
LTTLKVIDNAIGEDGARALADSTS
LTSLDARRNGIGEAGAQVLEANTR

Fig. 2. Structures of the GALA effector family. (a) Structures of the GALA effector family of $R$. solanacearum RS1000. Amino acid residue numbers are shown below the bars. Grey-shaded boxes indicate LRRs and the number is shown above. The black boxes indicate the F-box motif-like sequences. (b) Amino acid sequence of Hpx13/GALA6. The F-box motif-like sequences are underlined. LRR repeats are aligned, with numbers on the left. Conserved amino acids (more than $70 \%$ identity) in LRRs are indicated in blue bold letters. Amino acid residues that are substituted in $\mathrm{Hpx} 13$ compared with the corresponding protein (GALA6/RSc1356) in GMl1000 are shown in red.

proteins in the F-box motif-like sequences. Sequence comparison between the GALA effectors of our strain RS1000 and those of the genome-sequenced strain GMI1000 revealed that some members accumulate mutations in their LRR domain. Hyper-amino acid substitution was observed in LRRs of Hpx13 and its corresponding GALA6/RSc1356 of GMI1000; the percentage of amino acid substitution in the $\mathrm{N}$-terminal non-LRR region was $2.6 \%$ (5/190 amino acids), but in LRRs it was $27.5 \%$ (112/ 407 amino acids). Most of the amino acid substitutions in LRRs occurred in the variable residues but not in the conserved residues in the LRR motif (Fig. 2b). On the other hand, two independent deletion events, I ( $\Delta 3$ LRRs) and II ( $\triangle 4$ LRRs), occurred in the LRR domain of RSp0914/ GALA1 from RS1000 compared with that from GMI1000 (data not shown). These mutational changes in GALA effectors might contribute to the pathoadaptation of $R$. solanacearum strains to their host plants.

Eight of the remaining 18 effector proteins from $R$. solanacearum share homology with known type III effectors and enzymes (Mukaihara et al., 2004). The Hpx11, Hpx23, Hpx24, Hpx25 and Hpx27 effectors share homology with the HopQ1-1, HopAA1-1, HopR1, HopD1 and HopAV1 effectors from Pseudomonas syringae. Hpx17 is a member of the AvrBs3 effector family from xanthomonads. Hpx29 shares homology with the IpaH effector family from Shigella spp. It has been revealed how some of the abovementioned effectors function in eukaryotic host cells (Kay et al., 2007; Li et al., 2005; Munkvold, et al., 2008; Rohde et al., 2007; Wei et al., 2007). It would be interesting to determine whether $R$. solanacearum effectors function in a similar way. Hpx11 and Hpx26 have been found to possess known enzyme motifs, respectively called the inocineuridine-preferring nucleoside hydrolase motif and the Nudix (nucleoside diphosphates linked to some other moiety $\overline{\mathrm{X}}$ ) hydrolase motif (Mukaihara et al., 2004). Recently, it has been shown that an Arabidopsis Nudix family protein, AtNUDT7, negatively regulates the basal defence response through its nucleoside hydrolysis activity (Ge et al., 2007). Although such catalytic activities have not yet been determined in Hpx11 and Hpx26, these effectors might change the amount of certain nucleoside derivatives and modulate the defence response in plant cells.

It is noteworthy that, from a database search, many proteins similar to $R$. solanacearum effector proteins are distributed among plant-pathogenic bacteria, symbiotic bacteria and even in animal-pathogenic bacteria (data not shown). Although their abilities for translocation into host cells have not yet been clarified in most cases, they may also act as effector proteins during the infection of these bacteria. On the other hand, we could not find similar proteins for Нpx2, Нpx9, Нpx18, Нpx26, Нpx33, РopA and PopB effectors, indicating that they are specific to $R$. solanacearum.

Recent studies that focused on the entire type III secretome have revealed a large repertoire of effector proteins in plant- and animal-pathogenic bacteria. A nearly complete inventory analysis using multiple functional and bioinfor- 
matics-based approaches identified 41 functional type III effector proteins possessed by the phytopathogenic bacterium P. syringae pv. tomato DC3000 (Schechter et al., 2006). In animal pathogens, a similar approach revealed 39 functional effector proteins in enterohaemorrhagic $E$. coli O157 Sakai (Tobe et al., 2006). Considering these findings and the results obtained in our study, both plant- and animal-pathogenic bacteria seem to inject more than 40 effector proteins into host cells during infection. In this study, under the speculation that most effector genes of $R$. solanacearum are regulated by $h r p B$, we analysed 44 candidate effector genes. However, our search for $h r p B$ regulated genes is still ongoing (Mukaihara et al., 2004) and a genome-wide microarray analysis has resulted in the identification of $143 \mathrm{hrpB}$-upregulated genes in $R$. solanacearum GMI1000 (Occhialini et al., 2005). Therefore, a large number of untested genes potentially encoding effector proteins exist in $R$. solanacearum. It needs to be determined whether the remaining $h r p B$ upregulated genes that were not examined in this study encode effector proteins. As another approach in the search for effector proteins, several functional screening systems that can directly detect the in planta translocation activity of effector proteins have been constructed and used for some plant-pathogenic bacteria, such as $P$. syringae and Xanthomonas campestris (Chang et al., 2005; Guttman et al., 2002; Roden et al., 2004). It will also be important to construct such a functional screening system for the complete inventory of type III effector proteins in $R$. solanacearum.

\section{ACKNOWLEDGEMENTS}

This work was supported in part by a Grant-in-Aid for Scientific Research (No. 20580050) to T. M. from the Ministry of Education, Culture, Sports, Science and Technology, Japan.

\section{REFERENCES}

Alfano, J. R. \& Collmer, A. (1997). The type III (Hrp) secretion pathway of plant pathogenic bacteria: trafficking harpins, Avr proteins and death. J Bacteriol 179, 5655-5662.

Alfano, J. R. \& Collmer, A. (2004). Type III secretion system effector proteins: double agents in bacterial disease and plant defense. Annu Rev Phytopathol 42, 385-414.

Angot, A., Peeters, N., Lechner, E., Vailleau, F., Baud, C., Gentzbittel, L., Sartorel, E., Genschik, P., Boucher, C. \& Genin, S. (2006). Ralstonia solanacearum requires F-box-like domain-containing type III effectors to promote disease on several host plants. Proc Natl Acad Sci U S A 103 , 14620-14625.

Arlat, M., Van Gijsegem, F., Huet, J. C., Pernollet, J. C. \& Boucher, C. A. (1994). PopA1, a protein which induces a hypersensitivity-like response on specific Petunia genotypes, is secreted via the Hrp pathway of Pseudomonas solanacearum. EMBO J 13, 543-553.

Bai, C., Sen, P., Hofman, K., Ma, L., Goeble, M., Harper, J. W. \& Elledge, S. J. (1996). SKP1 connects cell cycle regulators to the ubiquitin proteolysis machinery through a novel motif, the F-box. Cell 86, 263-274.
Boucher, C. A., Barberis, P. A., Trigalet, A. P. \& Demery, D. (1985). Transposon mutagenesis of Pseudomonas solanacearum: isolation of Tn5-induced avirulent mutants. Microbiology 131, 2449-2457.

Boucher, C. A., Van Gijsegem, F., Barberies, P. A., Arlat, M. \& Zischek, C. (1987). Pseudomanas solanacearum genes controlling both pathogenicity on tomato and hypersensitivity on tobacco are clustered. J Bacteriol 169, 5626-5633.

Casper-Lindley, C., Dahlbeck, D., Clark, E. T. \& Staskawicz, B. J. (2002). Direct biochemical evidence for type III secretion-dependent translocation of the AvrBs2 effector protein into plant cells. Proc Natl Acad Sci U S A 99, 8336-8341.

Chang, J. H., Urbach, J. M., Law, T. F., Arnold, L. W., Hu, A., Gombar, S., Grant, S. R., Ausubel, F. M. \& Dangl, J. L. (2005). A high-throughput, near saturating screen for type III effector genes from Pseudomonas syringae. Proc Natl Acad Sci U S A 102, 2549-2554.

Cornelis, G. R. \& Van Gijsegem, F. (2000). Assembly and function of type III secretory systems. Annu Rev Microbiol 54, 735-774.

Cunnac, S., Occhialini, A., Barberis, P., Boucher, C. \& Gennin, S. (2004). Inventory and functional analysis of the large Hrp regulon in Ralstonia solanacearum: identification of novel effector proteins translocated to plant host cells through the type III secretion system. Mol Microbiol 53, 115-128.

Ferreira, A. O., Myers, C. R., Gordon, J. S., Martin, G. B., Vencato, M., Collmer, A., Wehling, M. D., Alfano, J. R., Moreno-Hagelsieb, G. \& other authors (2006). Whole-genome expression profiling defines the HrpL regulon of Pseudomonas syringae pv. tomato DC3000, allows de novo reconstruction of the Hrp cis element, and identifies novel coregulated genes. Mol Plant Microbe Interact 19, 1167-1179.

Fouts, D. E., Abramovitch, R. B., Alfano, J. R., Baldo, A. M., Buell, C. R., Cartinhour, S., Chatterjee, A. K., D'Ascenzo, M., Gwinn, M. L. \& other authors (2002). Genomewide identification of Pseudomonas syringae pv. tomato DC3000 promoters controlled by the HrpL alternative sigma factor. Proc Natl Acad Sci U S A 99, 2275-2280.

Galán, J. E. \& Collmer, A. (1999). Type III secretion machines: bacterial devices for protein delivery into host cells. Science 284, 13221328.

Galán, J. E. \& Wolf-Watz, H. (2006). Protein delivery into eukaryotic cells by type III secretion machines. Nature 444, 567-573.

Ge, X., Li, G.-J., Wang, S.-B., Zhu, H., Zhu, T., Wang, X. \& Xia, Y. (2007). AtNUDT7, a negative regulator of basal immunity in Arabidopsis, modulates two distinct defense response pathways and is involved in maintaining redox homeostasis. Plant Physiol 145, 204215.

Genin, S., Gough, C. L., Zischek, C. \& Boucher, C. A. (1992). Evidence that the $h r p B$ gene encodes a positive regulator of pathogenicity genes from Pseudomonas solanacearum. Mol Microbiol 6, 3065-3076.

Gophna, U., Ron, E. Z. \& Graur, D. (2003). Bacterial type III secretion systems are ancient and evolved by multiple horizontal-transfer events. Gene 312, 151-163.

Guéneron, M., Timmers, A. C. J., Boucher, C. A. \& Arlat, M. (2000). Two novel proteins, PopB, which has functional nuclear localization signals, and PopC, which has a large leucine-rich repeat domain, are secreted through the Hrp-secretion apparatus of Ralstonia solanacearum. Mol Microbiol 36, 261-277.

Guttman, D. S., Vinatzer, B. A., Sarkar, S. F., Ranall, M. V., Kettler, G. \& Greenberg, J. T. (2002). A functional screen for the type III (Hrp) secretome of the plant pathogen Pseudomonas syringae. Science 295, 1722-1726.

Hayward, A. C. (1991). Biology and epidemiology of bacterial wilt caused by Pseudomonas solanacearum. Annu Rev Phytopathol 29, 6587. 
He, S. Y., Nomura, K. \& Whittam, T. S. (2004). Type III protein secretion mechanism in mammalian and plant pathogens. Biochim Biophys Acta 1694, 181-206.

Jin, Q. \& He, S. Y. (2001). Role of the Hrp pilus in type III protein secretion in Pseudomonas syringae. Science 294, 2556-2558.

Jones, J. D. G. \& Dangle, J. L. (2006). The plant immune system. Nature 444, 323-329.

Kajava, A. V., Anisimova, M. \& Peeters, N. (2008). Origin and evolution of GALA-LRR, a new member of the CC-LRR subfamily: from plants to bacteria? PLoS ONE 3, e1694.

Kay, S., Hahn, S., Marois, E., Hause, G. \& Bonas, U. (2007). A bacterial effector acts as a plant transcription factor and induces a cell size regulator. Science 318, 648-651.

Li, X., Lin, H., Zhang, W., Zou, Y., Zhang, J., Tang, X. \& Zhou, J.-M. (2005). Flagellin induces innate immunity in nonhost interactions that is suppressed by Pseudomonas syringae effectors. Proc Natl Acad Sci U S A 102, 12990-12995.

Mudgett, M. B. (2005). New insights to the function of phytopathogenic bacterial type III effectors in plants. Annu Rev Plant Biol 56, 509-531.

Mukaihara, T., Tamura, N., Murata, Y. \& Iwabuchi, M. (2004). Genetic screening of Hrp type III-related pathogenicity genes controlled by the HrpB transcriptional activator in Ralstonia solanacearum. Mol Microbiol 54, 863-875.

Munkvold, K. R., Martin, M. E., Bronstein, P. A. \& Collmer, A. (2008). A survey of the Pseudomonas syringae pv. tomato DC3000 type III secretion system effector repertoire reveals several effectors that are deleterious when expressed in Saccharomyces cerevisiae. Mol Plant Microbe Interact 21, 490-502.

Murata, Y., Tamura, N. \& Mukaihara, T. (2006). Mutations in the $\operatorname{lrp} E$ gene of Ralstonia solanacearum affects Hrp pili production and virulence. Mol Plant Microbe Interact 19, 884-895.

Noël, L., Thieme, F., Nennstiel, D. \& Bonas, U. (2001). cDNA-AFLP analysis unravels a genome-wide $h r p G$-regulon in the plant pathogen Xanthomonas campestris pv. vesicatoria. Mol Microbiol 41, 1271-1281.

Nomura, K., Melotto, M. \& He, S. Y. (2005). Suppression of host defense in compatible plant-Pseudomonas syringae interactions. Curr Opin Plant Biol 8, 361-368.

Occhialini, A., Cunnac, S., Reymond, N., Genin, S. \& Boucher, C. (2005). Genome-wide analysis of gene expression in Ralstonia solanacearum reveals that the $h r p B$ gene acts as a regulatory switch controlling multiple virulence pathways. Mol Plant Microbe Interact 18, 938-949.

Petnicki-Ocwieja, T., Schneider, D. J., Tam, V. C., Chancey, S. T., Shan, L. T., Jamir, T., Schecter, L. M., Janes, M. D., Buell, C. R. \& other authors (2002). Genomewide identification of proteins secreted by the Hrp type III protein secretion system of Pseudomonas syringae pv. tomato DC3000. Proc Natl Acad Sci U S A 99, 7652-7657.

Roden, J. A., Belt, B., Ross, J. B., Tachibana, T., Vargas, J. \& Mudgett, M. B. (2004). A genetic screen to isolate type III effectors translocated into pepper cells during Xanthomonas infection. Proc Natl Acad Sci U $S$ A 101, 16624-16629.

Rohde, J. R., Breitkreutz, A., Chenal, A., Sansonetti, P. J. \& Parsot, C. (2007). Type III secretion effectors of the IpaH family are E3 ubiquitin ligases. Cell Host Microbe 1, 77-83.

Roine, E., Wei, W. S., Yuan, J., Nurmiaho-Lassila, E. L., Kalkkinen, N., Romantschuk, M. \& He, S. Y. (1997). Hrp pilus: a hrp-dependent bacterial surface appendage produced by Pseudomonas syringae pv. tomato DC3000. Proc Natl Acad Sci U S A 94, 3459-3464.

Salanoubat, M., Genin, S., Artiguenave, F., Gouzy, J., Mangenot, S., Arlat, M., Billault, A., Brottier, P., Camus, J. C. \& other authors (2002).
Genome sequence of the plant pathogen Ralstonia solanacearum. Nature 415, 497-502.

Sambrook, J., Fritsch, E. F. \& Maniatis, T. (1989). Molecular Cloning: a Laboratory manual, 2nd edn. Cold Spring Harbor, NY: Cold Spring Harbor Laboratory.

Schechter, L. M., Roberts, K. A., Jamir, Y., Alfano, J. R. \& Collmer, A. (2004). Pseudomonas syringae type III secretion system targeting signals and novel effectors studied with a Cya translocation reporter. J Bacteriol 186, 543-555.

Schechter, L. M., Vencato, M., Jordan, K. L., Schneider, S. E., Schneider, D. J. \& Collmer, A. (2006). Multiple approaches to a complete inventory of Pseudomonas syringae pv. tomato DC3000 type III secretion system effector proteins. Mol Plant Microbe Interact 19, 1180-1192.

Schell, M. A. (2000). Control of virulence and pathogenicity genes of Ralstonia solanacearum by an elaborate sensory network. Annu Rev Phytopathol 38, 263-292.

Simon, R., Priefer, U. \& Pühler, A. (1983). A broad host range mobilization system for in vivo genetic engineering: transposon mutagenesis in Gram negative bacteria. Biotechnology (N Y) 1, 784791.

Sory, M. P. \& Cornelis, G. R. (1994). Translocation of a hybrid YopEadenylate cyclase from Yersinia enterocolitica into HeLa cells. Mol Microbiol 14, 583-594.

Tamura, N., Murata, Y. \& Mukaihara, T. (2005). Isolation of Ralstonia solanacearum $h r p B$ constitutive mutants and secretion analysis of $h r p B$-regulated gene products that share homology with known type III effectors and enzymes. Microbiology 151, 2873-2884.

Tobe, T., Beatson, S. A., Taniguchi, H., Abe, H., Bailey, C. M., Fivian, A., Younis, R., Matthews, S., Marches, O. \& other authors (2006). An extensive repertoire of type III effectors in Escherichia coli O157 and the role of lambdoid phages in their dissemination. Proc Natl Acad Sci U S A 103, 14941-14946.

Van Gijsegem, F., Gough, C., Zischek, C., Niqueux, E., Arlat, M., Genin, S., Barberis, P., German, S., Castello, P. \& Boucher, C. (1995). The hrp locus of Pseudomonas solanacearum that controls the production of a type III secretion system, encodes eight proteins related to components of the bacterial flagellar biogenesis complex. Mol Microbiol 15, 1095-1114.

Van Gijsegem, F., Vasse, J., Camus, J. C., Marenda, M. \& Boucher, C. (2000). Ralstonia solanacearum produces Hrp-dependent pili that are required for PopA secretion but not for attachment of bacteria to plant cells. Mol Microbiol 36, 249-260.

Weber, E., Ojanen-Reuhs, T., Huguet, E., Hause, G., Romantschuk, M., Korhonen, T. K., Bonas, U. \& Koebnik, R. (2005). The type IIIdependent Hrp pilus is required for productive interaction of Xanthomonas campestris pv. vesicatoria with pepper host plants. J Bacteriol 187, 2458-2468.

Wei, C.-F., Kvitko, B. H., Shimizu, R., Crabill, E., Alfano, J. R., Lin, N. C., Martin, G. B., Huang, H.-C. \& Collmer, A. (2007). A Pseudomonas syringae pv. tomato DC3000 mutant lacking the type III effector HopQ1-1 is able to cause disease in the model plant Nicotiana benthamiana. Plant J 51, 32-46.

Zwiesler-Vollick, J., Plovanich-Jones, A., Nomura, K., Bandyopadhyay, S., Joardar, V., Kunkel, B. N. \& He, S. Y. (2002). Identification of novel $h r p$-regulated genes through functional genomic analysis of the Pseudomonas syringae pv. tomato DC3000 genome. Mol Microbiol 45, 1207-1218.

Edited by: I. K. Toth 